\title{
Low expression of NSD1, NSD2, and NSD3 define a subset of human papillomavirus- positive oral squamous carcinomas with unfavorable prognosis
}

Steven F. Gameiro ${ }^{1 \dagger}$, Farhad Ghasemi ${ }^{2+}$, Peter Y. F. Zeng ${ }^{3}$, Neil Mundi ${ }^{3}$, Christopher J. Howlett ${ }^{4}$, Paul Plantinga ${ }^{4}$, John W. Barrett ${ }^{3}$, Anthony C. Nichols ${ }^{3,4,5,6,7^{*}}$ and Joe S. Mymryk ${ }^{1,3,5,6,8^{*}}$ (iD

\begin{abstract}
Background: Frequent mutations in the nuclear receptor binding SET domain protein 1 (NSD1) gene have been observed in head and neck squamous cell carcinomas (HNSCC). NSD1 encodes a histone 3 lysine-36 methyltransferase. NSD1 mutations are correlated with improved clinical outcomes and increased sensitivity to platinum-based chemotherapy agents in human papillomavirus-negative (HPV-) tumors, despite weak T-cell infiltration. However, the role of NSD1 and related family members NSD2 and NSD3 in human papillomaviruspositive $(\mathrm{HPV}+)$ HNSCC is unclear.
\end{abstract}

Methods: Using data from over 500 HNSCC patients from The Cancer Genome Atlas (TCGA), we compared the relative level of mRNA expression of NSD1, NSD2, and NSD3 in HPV+ and HPV- HNSCC. Correlation analyses were performed between T-cell infiltration and the relative level of expression of NSD1, NSD2, and NSD3 mRNA in HPV+ and HPV- HNSCC. In addition, overall survival outcomes were compared for both the HPV+ and HPV- subsets of patients based on stratification by NSD1, NSD2, and NSD3 expression levels.

Results: Expression levels of NSD1, NSD2 or NSD3 were not correlated with altered lymphocyte infiltration in HPV+ HNSCC. More importantly, low expression of NSD1, NSD2, or NSD3 correlated with significantly reduced overall patient survival in HPV+, but not HPV- HNSCC.

Conclusion: These results starkly illustrate the contrast in molecular features between HPV+ and HPV- HNSCC tumors and suggest that NSD1, NSD2, and NSD3 expression levels should be further investigated as novel clinical metrics for improved prognostication and patient stratification in HPV+ HNSCC.

Keywords: Head and neck cancer, Head and neck squamous cell carcinoma, HPV, WHSC1, WHSC1L1, Epigenetics, Histone methyltransferase, The Cancer Genome Atlas

\footnotetext{
* Correspondence: Anthony.Nichols@lhsc.on.ca; jmymryk@uwo.ca

†Steven F. Gameiro and Farhad Ghasemi contributed equally to this work.

${ }^{3}$ Department of Otolaryngology, Head \& Neck Surgery, The University of Western Ontario, London, ON N6A 3K7, Canada

${ }^{1}$ Department of Microbiology and Immunology, The University of Western Ontario, London, ON N6A 3K7, Canada

Full list of author information is available at the end of the article
}

(c) The Author(s). 2021 Open Access This article is licensed under a Creative Commons Attribution 4.0 International License, which permits use, sharing, adaptation, distribution and reproduction in any medium or format, as long as you give appropriate credit to the original author(s) and the source, provide a link to the Creative Commons licence, and indicate if changes were made. The images or other third party material in this article are included in the article's Creative Commons licence, unless indicated otherwise in a credit line to the material. If material is not included in the article's Creative Commons licence and your intended use is not permitted by statutory regulation or exceeds the permitted use, you will need to obtain permission directly from the copyright holder. To view a copy of this licence, visit http://creativecommons.org/licenses/by/4.0/ The Creative Commons Public Domain Dedication waiver (http://creativecommons.org/publicdomain/zero/1.0/) applies to the data made available in this article, unless otherwise stated in a credit line to the data. 


\section{Introduction}

Head and neck squamous cell carcinomas (HNSCC) comprise a group of heterogeneous cancers that arise from multiple anatomical subsites in the head and neck region. Collectively, they represent the 7th most common human cancer type [1] and are often characterized by aggressive local invasion and overall poor prognosis [2]. Historically, tobacco use and alcohol consumption are major risk factors for HNSCC [3]. However, infection with human papillomavirus (HPV) has recently emerged as a major cause of tumors located in the oropharynx. Indeed, HPV-positive (HPV+) HNSCC is increasing at an epidemic rate $[4,5]$. Numerous studies have confirmed that HPV-negative (HPV-) and HPV+ HNSCC are molecularly distinct [6]. HPV+ HNSCC constitutively express the viral $E 6$ and $E 7$ oncogenes that deregulate cell growth and gene expression [7], at least in part via epigenetic mechanisms [8, 9]. Importantly, clinical outcomes for HPV+ HNSCC are superior to those of HPV- cases [10, 11], allowing for potential stratification of patients into alternative treatment regimens based on HPV status [12].

Histone methylation plays a critical role in the epigenetic control of gene expression. Specialized enzymes methylate/de-methylate individual amino acids that are found on the histone tails $\mathrm{H} 1, \mathrm{H} 2 \mathrm{~A}, \mathrm{H} 2 \mathrm{~B}, \mathrm{H} 3$, and $\mathrm{H} 4$. The nuclear receptor binding SET domain protein (NSD) family of histone-lysine N-methyltransferases are composed of three paralogous proteins: NSD1, NSD2 (WHSC1), and NSD3 (WHSC1L1). NSD1 mediates the transfer of a methyl group onto H3 lysine-36 (H3K36) and H4 lysine-20 (H4K20) [13]. NSD2 methylates H3 lysine-4 (H3K4) and H4K20, and NSD3 methylates H3K36 [14]. NSD paralogs also methylate non-histone substrates, these include NSD1-mediated methylation of nuclear factor kappa-light-chain-enhancer of activated B cells (NFкB) [15]; NSD2-mediated methylation of phosphatase and tensin homolog (PTEN) [16]; and NSD3-mediated methylation of interferon regulatory factor 3 (IRF3) and epidermal growth factor receptor (EGFR) [17, 18]. Furthermore, a functional role for NSD2 in the type-I interferon response has also been reported [19]. Moreover, numerous studies have linked the NSD family of methyltransferases with a variety of different cancers [20].

We and others used hierarchical clustering of DNA methylation data to identify a subset of HPV- HNSCC tumors enriched for mutations in the NSD1 H3K36 methyltransferase $[8,9]$. These cluster with tumors expressing wild-type NSD1 that contain a substitution of K36 to methionine in H3. Thus, mutation of the substrate of NSD1 phenocopies the methylation signature of direct mutational activation of this enzyme. This signature represents approximately 13\% of HPV- HNSCC and identifies NSD1 inactivation as a mechanism of epigenome deregulation [8]. Studies using multiple cohorts have demonstrated that HPV- HNSCC patients with NSD1 gene alterations exhibited improved survival compared to patients with wild-type NSD1 tumors [21, 22]. Furthermore, mutation or reduced expression of NSD1 in HPV- HNSCC has been reported to confer increased sensitivity to platinum-based chemotherapy agents in vitro $[22,23]$, despite leading to an immunologically "cold" phenotype characterized by slightly reduced T-cell infiltration [24]. Another study suggested that mutation of NSD1 or NSD2 leads to significantly better clinical outcomes in HPV- HNSCC of the larynx [25], further supporting an oncogenic role for these methyltransferases in HPV- HNSCC. In contrast, little is known about the role of $N S D 1$, or its paralogs $N S D 2$ and NSD3 in HPV+ HNSCC, except that mutation of these genes appears to occur at a reduced frequency as compared to HPV- HNSCC $[6,26]$ and NSD1 mutation is correlated with reduced survival [22].

In this study, we used data from over 500 HNSCC patients from the Cancer Genome Atlas (TCGA) to compare the expression levels of mRNA for NSD1, NSD2, and NSD3 between HPV+ HNSCC, HPV- HNSCC, or normal control tissues. We also investigated the relationship between the levels of expression of NSD1, NSD2, or NSD3 with tumor-infiltrating lymphocytes (TILs) in either the HPV+ or HPV- HNSCC samples and whether expression of NSD1, NSD2, or NSD3 correlated with overall survival. Our aim was to determine if there were differences in these molecular features that could serve as novel clinical metrics for improved prognostication and patient stratification in HNSCC.

\section{Material and methods Data collection}

Patient data from the Cancer Genome Atlas (TCGA), including the Merged Clinical data and Level 3 RNA-Seq by Expectation-Maximization (RSEM) normalized Illumina HiSeq RNA expression data for the HNSCC cohort, was downloaded from the Broad Genome Data Analysis Centers Firehose server (https://gdac. broadinstitute.org/). RNA-seq viral read counts for HPV $E 6$ and $E 7$ was extracted from the supplementary data files from Chakravarthy et al. [27]. Patient survival data for the TCGA HNSCC cohort was extracted from the Pan-Cancer Clinical Data Resource [28]. All data utilized in this study can be found in Additional file 1 - Supplementary Table 1 (Table S1).

\section{RNA expression comparisons}

RSEM normalized expression data was extracted and curated as described [29]. Primary patient samples with known HPV status were grouped as HPV+, HPV-, or normal control tissues. This resulted in $73 \mathrm{HPV}+, 442$ 
HPV-, and 43 matched normal-adjacent control samples with data available for the HNSCC gene expression analysis. Boxplot comparisons of gene expression was performed using GraphPad Prism v7.0 (Graphpad Software, Inc., San Diego, California, USA) and assembled into final form using Adobe Illustrator (Adobe Systems Inc., San Jose, CA, USA). For the boxplots, center lines show the medians, box limits indicate the 25th and 75th percentiles as determined by Graphpad Prism and whiskers extend 1.5 times the interquartile range from the 25th and 75 th percentiles. Statistical significance was calculated using Graphpad Prism v7.0. The statistical $p$ values were assigned using a two-tailed non-parametric MannWhitney U test.

\section{Correlation matrix}

Level 3 RSEM normalized RNA-seq data for NSD1, NSD2, and NSD 3 were extracted from the TCGA database and processed into HPV+ and HPV- cohorts as detailed above. As mentioned above, HPV E6 and E7 RNA expression data was extracted from Chakravarthy et al. [27]. A pairwise Spearman correlation was performed for each of the aforementioned genes. Correlations were performed using RStudio (version 1.2.1335) utilizing the ggplot2 package [30]. The final correlation matrix figure was assembled using Adobe Illustrator (Adobe Systems Inc., San Jose, CA, USA).

\section{Survival analyses}

RSEM normalized RNA-seq data for each of NSD1, NSD2, and NSD3 were converted into a standard score (zscore) and grouped into high, mid, and low expression based on the following criteria: high $=\mathrm{z}$-score $>0.5$, mid $=-0.5<\mathrm{z}$-score $<0.5$, and low $=\mathrm{z}$-score $<-0.5$. Fiveyear overall survival outcomes were compared in both $\mathrm{HPV}+$ and HPV- subsets of patients grouped by either high, mid, or low expression of NSD1, NSD2, or NSD3. Log-rank statistical analysis was performed using GraphPad Prism v7.0 (Graphpad Software, Inc., San Diego, California, USA). Furthermore, log-rank $p$ values were assessed for significance after correcting for false discovery rate (FDR) using the Benjamini-Hochberg method with an FDR threshold of 10\%. Figures were assembled into final form using Adobe Illustrator (Adobe Systems Inc., San Jose, CA, USA). Univariate analysis was performed through RStudio (version 1.2.1335) based on a Cox Proportional-Hazards Model with the survival package (version 2.41-3). Finally, stepwise bidirectional multivariate analysis was performed with the grouped expression of the NSD paralogs and the following clinical variables: sex, age, subsite, $\mathrm{T}$ stage, $\mathrm{N}$ stage, overall stage, HPV type, and smoking history. The smoking history clinical variable for the HPV+ cohort was stratified as heavy smokers (>20 pack year history) or non- smokers based on our previous study that employed a similar stratification approach [21]. Moreover, patients with no smoking history information, patients with between 1 and 20 pack years, and patients who were listed as current or former smokers but with unknown pack year history were excluded. Statistical $p$ values were derived from the Wald test on survival coefficients.

\section{Correlation analysis for T-cell infiltration status}

To estimate T-cell infiltration, we used a previously generated T-cell signature based on mean expression of 13 transcripts [31] as utilized by Brennan et al. [24]. The expression of NSD1, NSD2, and NSD3 relative to that of the T-cell infiltration signature were compared in a pairwise fashion and concordance calculated by Spearman's Rho analysis.

\section{Results}

Expression of NSD1, NSD2, and NSD3 in HNSCC stratified by HPV status

Given that NSD1 and NSD2 have been linked to various aspects of HPV- HNSCC, including epigenetic alterations, immune status of the tumor, and predicting patient outcome at specific subsites $[8,9,21-26]$, we investigated the roles of NSD1 and its two paralogs NSD2 and NSD3 in HPV + HNSCC. Previous work has suggested that NSD1 RNA expression can serve as a measure of NSD1 proficiency in HNSCC [24]. As such, we analyzed the TCGA Illumina HiSeq RNA expression data from the HNSCC cohort for expression of all three paralogous genes (Fig. 1). Unexpectedly, the HPV+ samples had significantly increased levels of expression of all three paralogs as compared to HPV- tumors and normal control tissues.

To determine if this significant increase in expression of NSD paralogs in the HPV + cohort was due to the constitutive expression of viral oncogenes, we correlated HPV E6 and E7 mRNA expression levels with those of NSD1, $N S D 2$, or NSD3. Our analysis revealed a statistically significant positive Spearman correlation coefficient between NSD2 expression and either E6 or E7 (Fig. 2). In contrast, the Spearman correlation coefficients between the HPV viral oncogenes and NSD1 or NSD3 were not statistically significant (Fig. 2). Thus, the HPV viral oncogenes and NSD2 have an increasing monotonic relationship that may explain the high expression observed for NSD2 in the $\mathrm{HPV}+\mathrm{HNSCC}$ cohort compared to its HPV- counterpart and normal control tissues. Alternatively, E6 and E7 expression could be regulated by NSD 2 .

\section{HNSCC tumor samples concordantly express NSD1, NSD2, and NSD3}

Given that all three NSD paralogs are expressed on average at significantly higher levels in HPV + HNSCC, but only NSD2 had a statistically significant positive 


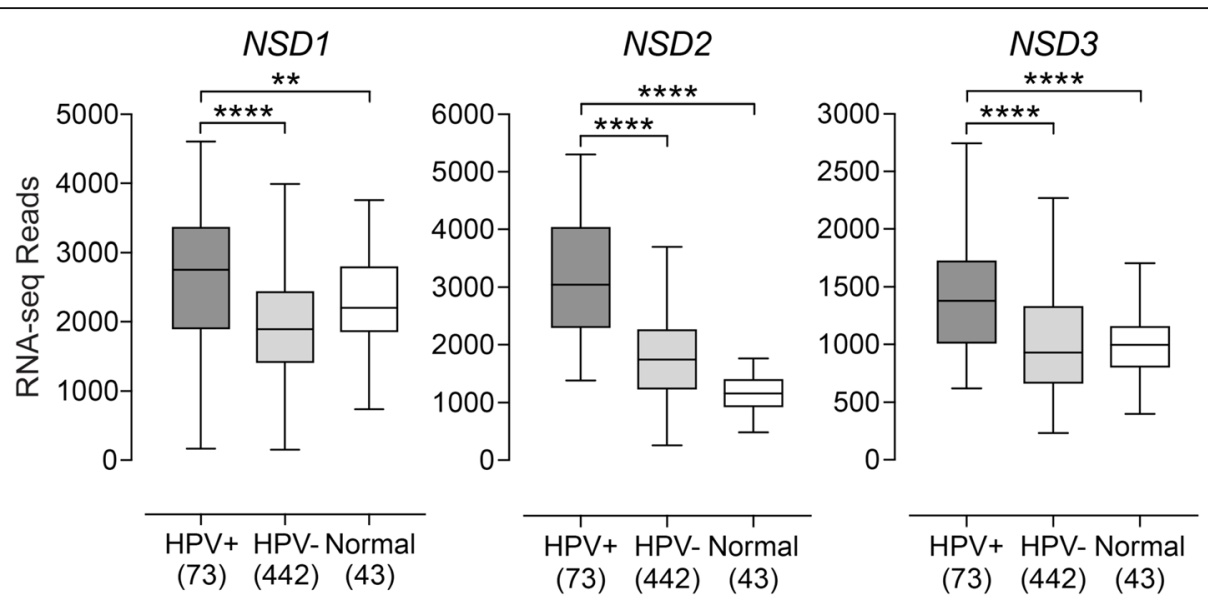

Fig. 1 Expression of NSD1, NSD2, and NSD3 genes in HNSCC stratified by HPV status. Normalized RNA-seq data was extracted from TCGA database for the HNSCC cohort for HPV+, HPV-, and matched normal-adjacent control tissues. Numbers in brackets refer to the number of samples included in each analysis. Statistical $p$ value significance levels are indicated as follows: ${ }^{*} p \leq 0.05 ;{ }^{* *} p \leq 0.01 ;{ }^{* * *} p \leq 0.001$; **** $p \leq$ 0.0001 ; ns - not significant

Spearman correlation coefficient with the HPV oncogenes, we next wanted to determine if individual tumors expressed high levels of a single NSD paralog on a mutually exclusive basis, or if their expression might be coordinately regulated. We performed pairwise analysis of RNA expression of each paralog with respect to the others for each sample in the HPV+ and HPV- subsets of this cohort (Fig. 2). This pairwise analysis indicated that HPV+ tumors expressing a high level of one para$\log$ express higher relative levels of the other paralogs. Similarly, HPV+ tumors expressing a low level of one paralog express lower relative levels of the other paralogs. A similar correlation was observed for HPV- tumors. Thus, coordinately upregulated expression of all three NSD paralogs is frequently observed in HNSCC, regardless of HPV status.

\section{T-cell infiltration is correlated with NSD1 expression in HPV-, but not HPV+ HNSCC}

Despite improved prognosis, NSD1 mutation in HPVHNSCC has been associated with an immunologically "cold" tumor microenvironment associated with slightly

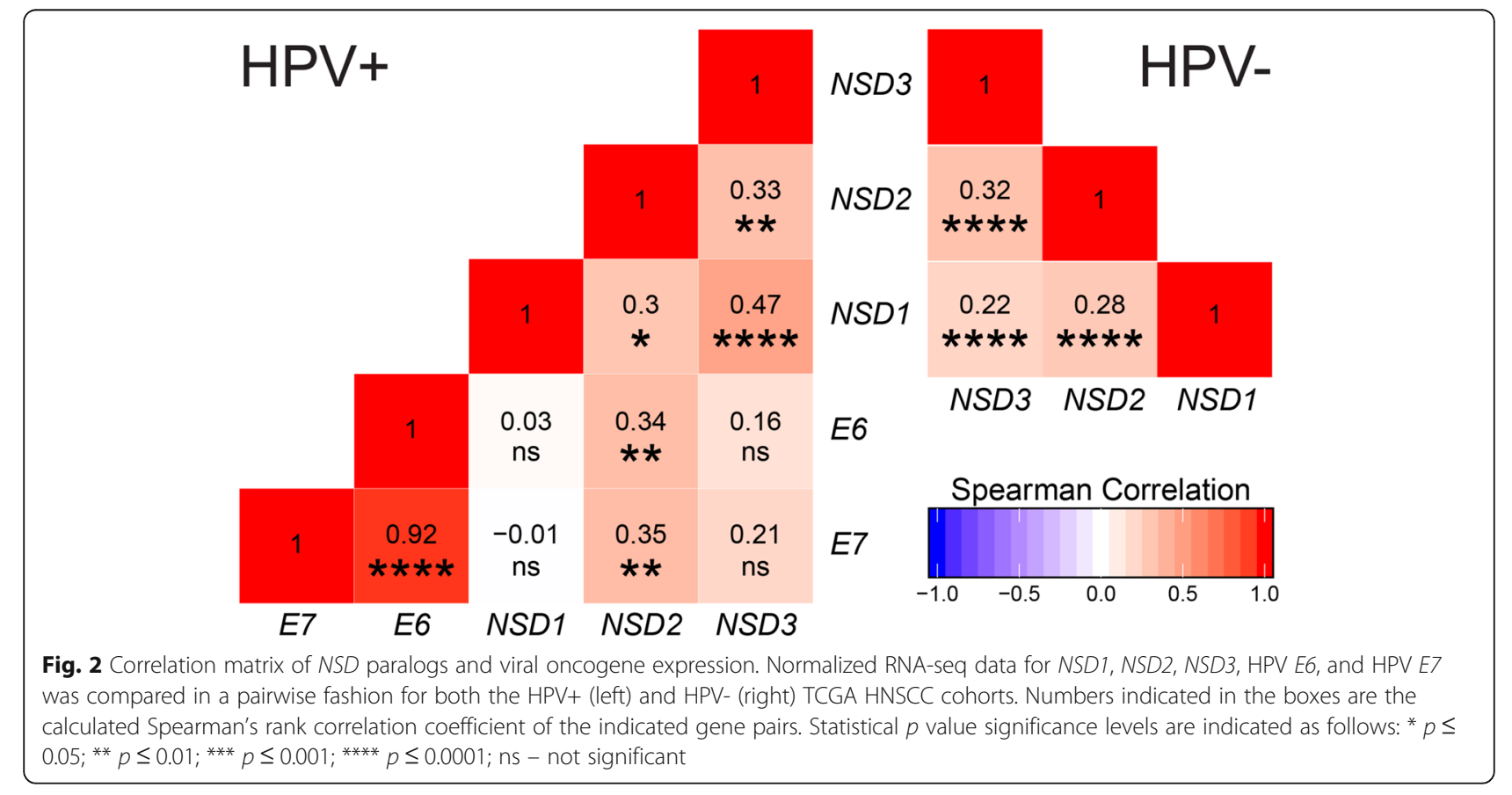


low levels of TILs [24]. Furthermore, weak T-cell infiltration was also associated with decreased NSD1 expression in other patient-derived datasets for HPV- tumors and experimental tumor models with HPV- HNSCC cells [24]. Utilizing the same 13 gene T-cell signature defined by Spranger et al. [31], T-cell infiltration was clearly higher in HPV+ HNSCC compared to the HPVcounterparts and normal control tissues (Fig. 3), consistent with the immunologically "hot" phenotype associated with HPV+ HNSCC [32, 33]. We next determined whether NSD1 levels were inversely associated with Tcell infiltration in HNSCC as reported previously for HPV- HNSCC [24]. We found no significant correlation between markers of $\mathrm{T}$-cell infiltration and expression of either NSD1, NSD2, or NSD3 in the HPV+ HNSCC cohort (Fig. 4a). In contrast, a weak but statistically significant positive Spearman correlation coefficient with NSD1 expression was observed for HPV- HNSCC (Fig. 4b), which is in agreement with the aforementioned study by Brennan et al. [24]. However, no statistically significant Spearman correlation coefficients were observed between either NSD2 or NSD3 expression and TIL score in the HPV- HNSCC cohort (Fig. 4b). Thus, a possible connection between NSD1 expression and T-cell infiltration into the tumor microenvironment appears unique to HPV-

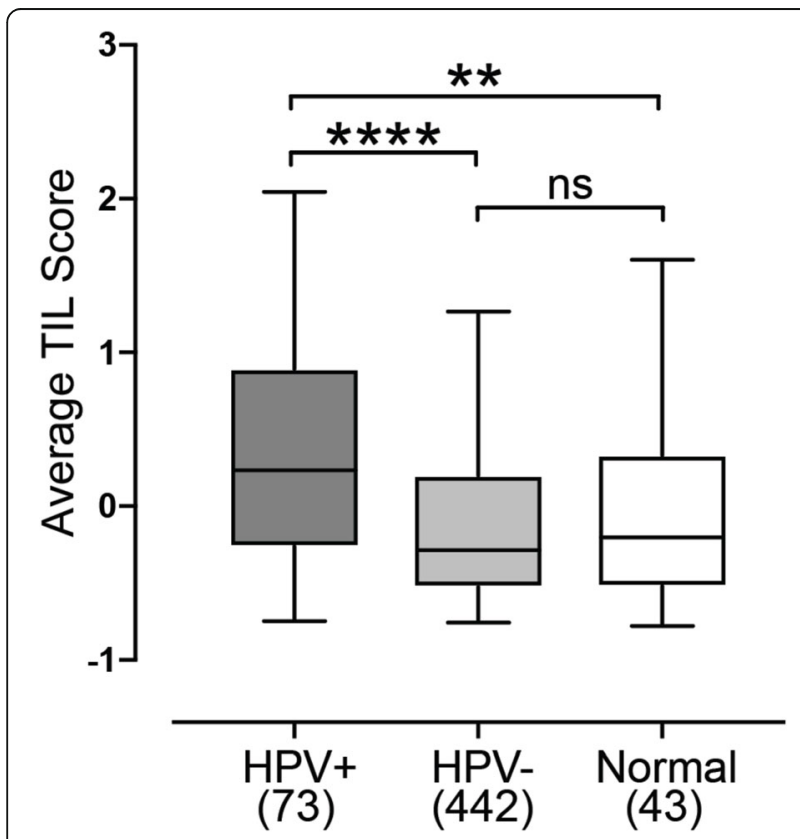

Fig. 3 T-cell infiltration is higher in HPV+ HNSCC compared to HPVand normal control counterparts. A previously reported 13 gene Tcell transcript signature was calculated for $\mathrm{HPV}+, \mathrm{HPV}$-, and matched normal-adjacent control tissues. Numbers in brackets refer to the number of samples included in each analysis. Statistical $p$ value significance levels are indicated as follows: * $p \leq 0.05 ;{ }^{* *} p \leq 0.01$; *** $p \leq 0.001 ;{ }^{* * * *} p \leq 0.0001$; ns - not significant
HNSCC samples and is not shared with the other NSD paralogs.

\section{Low expression levels of either NSD1, NSD2, or NSD3 predict reduced overall survival in HPV+ HNSCC}

Our previous study did not observe any significant relationship between the NSD1/H3K36M methylation signature and clinical outcomes in the TCGA HNSCC cohort [8]. More recently, the presence of damaging mutations in NSD1 or NSD2 have been linked to significantly improved patient survival, but only in HPV- tumors [21, 22, 25]. We subdivided the HPV+ and HPV- HNSCC dataset based on high $(\mathrm{z}$-score $>0.5)$, mid $(-0.5<\mathrm{z}$ score $<0.5$ ), or low (z-score $<-0.5)$ NSD1, NSD2, or NSD3 RNA expression and calculated the impact of expression on overall patient survival (Fig. 5). Unexpectedly, low expression of either NSD1, NSD2, or NSD3 predicted markedly reduced survival in HPV+ HNSCC over those patients with tumors expressing the NSD paralogs in the mid and high expression groups. Furthermore, this sharp overall decrease in clinical outcome was also statistically significant (Fig. 5a). In contrast, there was no statistically significant impact of either NSD1, NSD2, or NSD3 expression on overall survival for patients with HPV- HNSCC (Fig. 5b).

To determine the extent that each of the NSD family members could influence patient outcomes in the HPV+ HNSCC cohort, we generated a hazard ratio (HR) for each gene by univariate analysis (Table 1). As expected, the HRs for either NSD1 (low vs high expression and low vs mid expression), NSD2 (low vs high expression), and NSD3 (low vs high expression and low vs mid expression) were statistically significant, with NSD2 (low vs mid expression) trending towards significance. Since expression of these genes might not be independent predictors of survival, we analyzed the relationship between survival and our gene expression groups (high, mid, or low expression) for NSD1, NSD2, and NSD3 concurrently by multivariate analysis (Table 1 ). In addition to the expression of the NSD paralogs, we also included clinical variables that could influence outcome in our multivariate analysis, such as: sex, anatomical subsite, $\mathrm{T}$ stage, N stage, overall stage, HPV type, and smoking history (Table 1). The results of our multivariate analysis revealed that the HRs for NSD1 (low vs high expression) and NSD3 (low vs high expression and low vs mid expression) remained significant $(\mathrm{HR}=32.88,95 \%$ confidence interval $(\mathrm{CI})=3.23-333.33, p=0.003$; $\mathrm{HR}=103.1$, $95 \% \mathrm{CI}=8.55-1.24 \mathrm{e} 03, p=0.0003 ; \mathrm{HR}=135.3,95 \% \mathrm{CI}=$ $6.62-2.76 \mathrm{e} 03, p=0.001$, respectively), indicating that low expression of NSD1 and NSD3 is a significant, and potentially independent, contributor to overall survival in the HPV+ HNSCC cohort. The final multivariate model also included age $(\mathrm{HR}=0.13,95 \% \mathrm{CI}=0.023-0.70, p=$ 

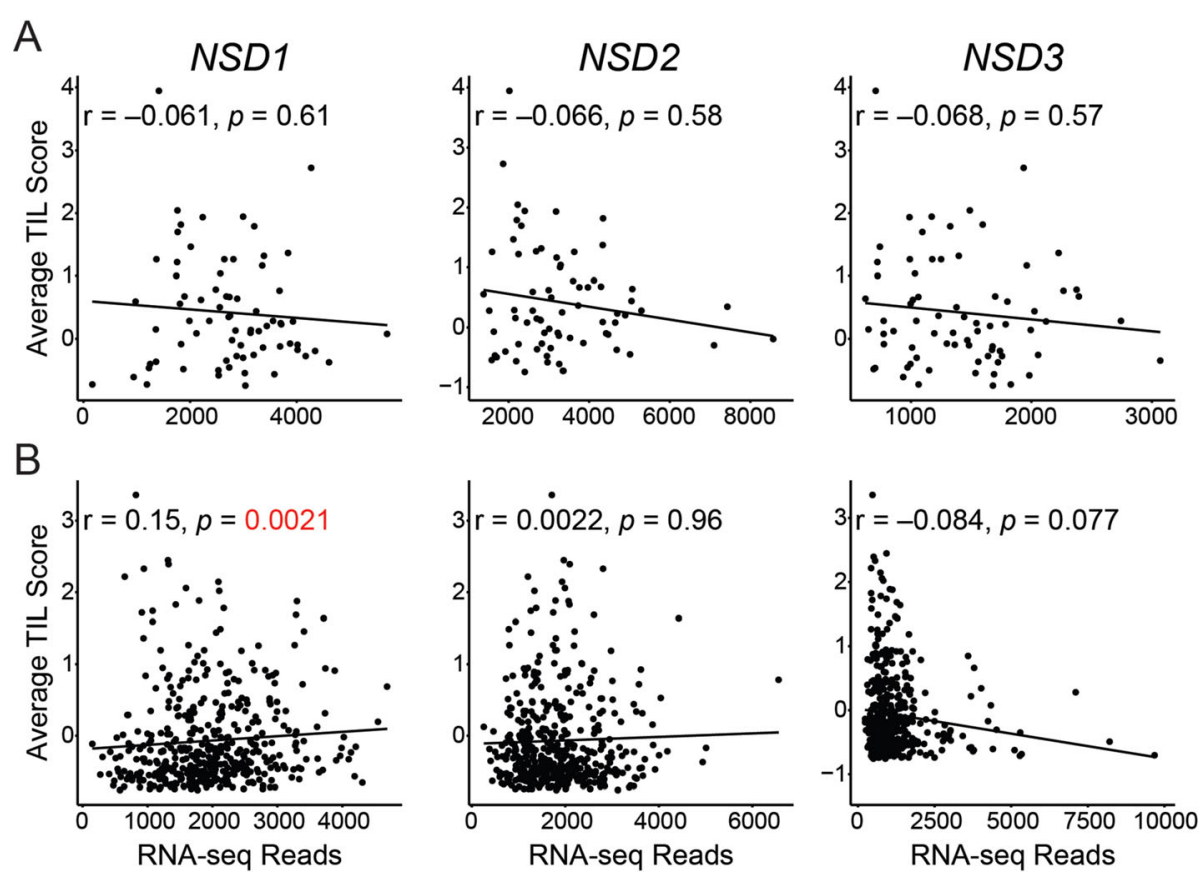

Fig. 4 T-cell infiltration is not correlated with NSD1 expression in HPV+ HNSCC, but is weakly correlated in HPV- HNSCC. A previously reported 13 gene T-cell transcript signature was calculated for each sample in the TCGA HNSCC cohort and compared pairwise with normalized RNA-seq data for NSD1, NSD2, and NSD3 for HPV+ (a) and HPV- (b) HNSCC samples. For each NSD paralog, the correlation with the T-cell transcript signature was calculated by Spearman's Rho analysis

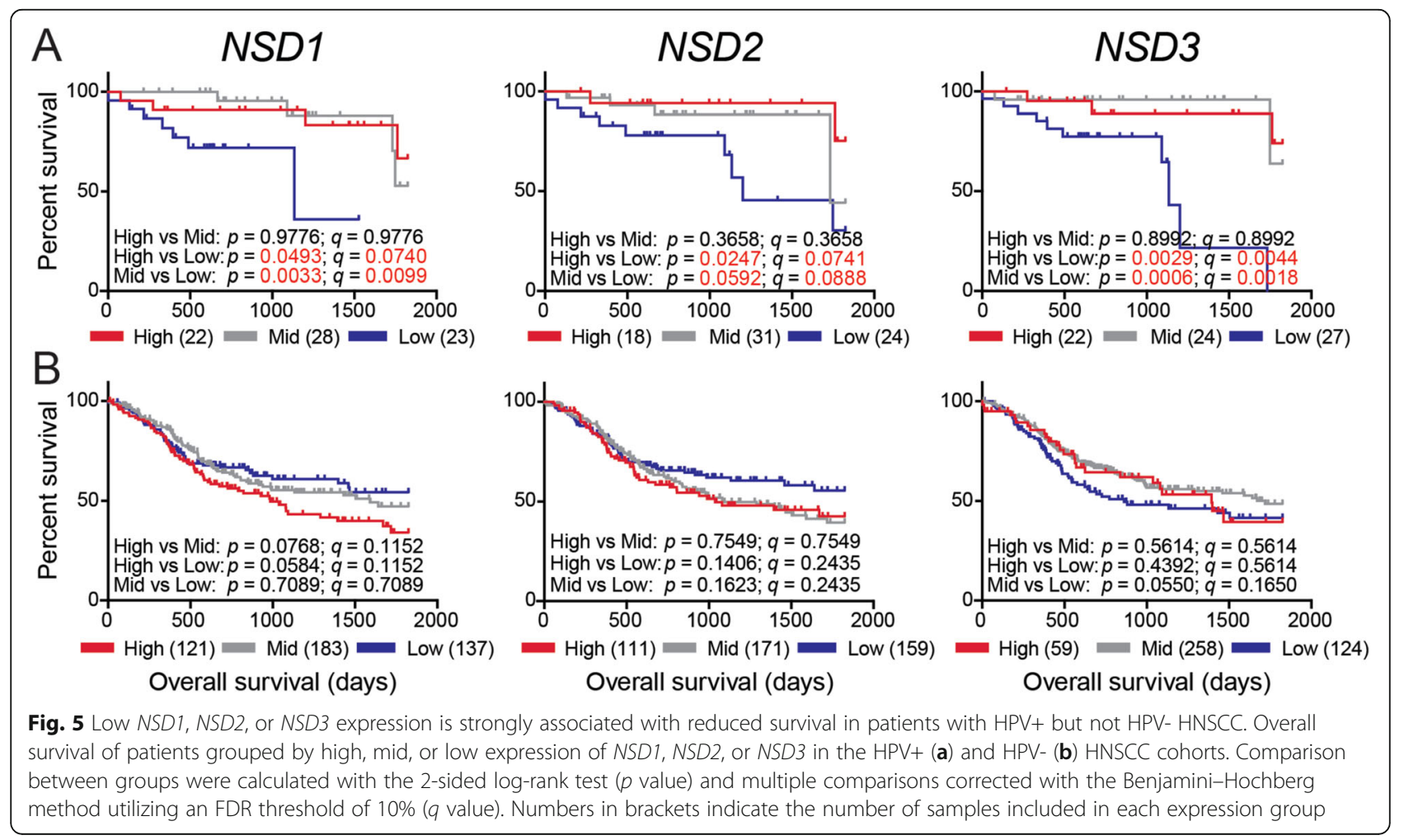


Table 1 Univariate and multivariate analysis of NSD paralog expression and clinical variables and their association with overall survival in the HPV+ HNSCC cohort

\begin{tabular}{|c|c|c|c|c|c|}
\hline \multirow[b]{2}{*}{ Variables } & & \multicolumn{2}{|l|}{ Univariate Analysis } & \multicolumn{2}{|l|}{ Multivariate Analysis } \\
\hline & & $\mathrm{HR}(95 \% \mathrm{Cl})$ & $P$ value & HR $(95 \% \mathrm{Cl})$ & $P$ value \\
\hline Sex & Male vs Female & $0.81(0.18-3.62)$ & 0.78 & & \\
\hline Age & $<60$ vs $\geq 60$ & $0.96(0.32-2.83)$ & 0.94 & $0.13(0.023-0.70)$ & 0.02 \\
\hline \multirow[t]{3}{*}{ Subsite } & Oral Cavity vs Oropharynx & $2.82(1.02-7.80)$ & 0.045 & $2.51(0.55-11.48)$ & 0.23 \\
\hline & Other vs Oropharynx & $1.54 \mathrm{e}-08(0-\operatorname{lnf})$ & 1.00 & $3.72 \mathrm{e}-10(0-\ln f)$ & 1.00 \\
\hline & Oral Cavity vs Other & $1.84 \mathrm{e} 08(0-\operatorname{lnf})$ & 1.00 & $6.75 \mathrm{e} 09(0-\ln f)$ & 1.00 \\
\hline T Stage & $\mathrm{T} 3-\mathrm{T} 4$ vs $\mathrm{T} 1-\mathrm{T} 2$ & $1.03(0.36-2.91)$ & 0.96 & & \\
\hline N Stage & $\mathrm{N} 2 \mathrm{~b}-\mathrm{N} 3$ vs N0 - N2a & $0.41(0.14-1.19)$ & 0.10 & $0.19(0.034-1.047)$ & 0.06 \\
\hline Overall Stage & $\mid V$ vs I - III & $0.76(0.26-2.24)$ & 0.62 & $0.034(0.004-0.33)$ & 0.003 \\
\hline HPV Type & Other vs 16 & $3.33(1.14-9.78)$ & 0.028 & $13.03(2.46-69.13)$ & 0.003 \\
\hline Smoking History & Heavy Smoker vs Non Smoker & $1.57(0.47-5.22)$ & 0.47 & & \\
\hline \multirow[t]{3}{*}{ NSD1 } & Low vs High Expression & $6.15(1.43-26.32)$ & 0.015 & $32.88(3.23-333.33)$ & 0.003 \\
\hline & Low vs Mid Expression & $5.95(1.49-23.81)$ & 0.012 & $4.94(0.84-29.41)$ & 0.08 \\
\hline & High vs Mid Expression & $0.97(0.24-3.91)$ & 0.96 & $0.15(0.018-1.23)$ & 0.08 \\
\hline \multirow[t]{3}{*}{ NSD2 } & Low vs High Expression & $5.08(1.08-23.88)$ & 0.04 & & \\
\hline & Low vs Mid Expression & $2.93(0.89-9.67)$ & 0.08 & & \\
\hline & High vs Mid Expression & $0.58(0.10-3.29)$ & 0.54 & & \\
\hline \multirow[t]{3}{*}{ NSD3 } & Low vs High Expression & $10.03(1.98-50.94)$ & 0.005 & $103.1(8.55-1.24 \mathrm{e} 03)$ & 0.0003 \\
\hline & Low vs Mid Expression & $10.63(2.03-55.62)$ & 0.005 & 135.3 (6.62-2.76e03) & 0.001 \\
\hline & High vs Mid Expression & $1.06(0.17-6.63)$ & 0.95 & $1.31(0.12-14.34)$ & 0.82 \\
\hline
\end{tabular}

$P<0.05$ are in italic

0.02), overall stage $(\mathrm{HR}=0.034,95 \% \mathrm{CI}=0.004-0.33$, $p=0.003)$, and HPV type ( $\mathrm{HR}=13.03,95 \% \mathrm{CI}=2.46-$ $69.13, p=0.003)$ as statistically significant contributing factors to survival.

\section{Discussion}

In general, the clinical management of HNSCC is complex, often associated with significant treatment induced morbidities and associated with unacceptably low clinical outcomes. HPV-dependent tumors of the oropharynx are a notable exception, as this subset of HNSCC exhibits dramatically better clinical outcomes. As a result, there is an effort to de-intensify treatment for patients with $\mathrm{HPV}+$ disease in an effort to reduce the acute and chronic toxicities associated with the aggressive treatment protocols necessary for treatment of HPV- HNSCC [12]. Nevertheless, approximately 15$20 \%$ of patients with $\mathrm{HPV}+$ disease still fail treatment. The cause of their treatment failure is still unknown, although there is some evidence that outcome depends on HPV genotype and clinical parameters such as tumor size and patient smoking history [10, 34, 35]. However, additional prognostic biomarkers that accurately predict the level of therapeutic intensity necessary for effective treatment are urgently needed.
A variety of evidence indicates that the NSD1 methyltransferase gene and its paralogs are actively involved in the development of a subset of HNSCC [8] and that their mutation status can predict clinical outcome in HPV- HNSCC [21, 25]. Using TCGA data, we found that $\mathrm{HPV}+$ tumors expressed statistically significant higher levels of NSD1, NSD2, and NSD3 compared to their HPV- counterparts and normal control tissues (Fig. 1). The unexpected observation that all three paralogs were upregulated in the $\mathrm{HPV}+$ cohort could be related to the constitutive expression of the viral E6 and $E 7$ oncogenes. Correlation of the expression of $E 6$ and $E 7$ mRNA with those of NSD1, NSD2, or NSD3 in a pairwise fashion indicated that only NSD2 had a statistically significant correlation with either E6 or E7 (Fig. 2). We also investigated if individual tumor samples expressed high levels of a single NSD paralog on a mutually exclusive basis, or if their expression might be coordinately regulated. Our pairwise analysis indicated that tumors expressing high levels of one of these methyltransferases expressed high levels of the other two paralogs (Fig. 2). This coordinate expression pattern was observed in both the HPV+ and HPV- HNSCC cohorts. Thus, coordinate upregulation of the NSD paralogs does not appear to be a specific consequence of HPV oncogene expression, as it is observed in both HPV+ and HPV-tumors. 
One study reported that mutations in NSD1, or low NSD1 expression leads to an immune "cold" phenotype in HPV- HNSCC [24]. This was characterized using a 13 gene TIL signature developed from melanoma studies [31]. As HPV+ HNSCC are generally considered immune "hot" tumors, with higher immune infiltration and CD8+ T-cell activation compared to HPV- HNSCC [32], we tested whether expression levels of the NSD paralogs was also linked to TIL levels in HPV+ samples. Despite confirming a weak correlation between NSD1 RNA expression and TIL signature in HPV- HNSCC (Fig. 4b), and confirming that the TIL signature is generally higher in HPV+ HNSCC compared to both HPV- HNSCC and normal control tissues (Fig. 3), no significant correlation was observed for NSD1, NSD2, or NSD3 in HPV+ HNSCC (Fig. 4a). Thus, expression of the NSD paralogs is not correlated with the infiltrating $\mathrm{T}$-cell component of the tumor microenvironment in HPV+ HNSCC. This illustrates yet another difference between $\mathrm{HPV}+$ and HPV- HNSCC. Interestingly, no significant relationship between NSD2 or NSD3 expression with the TIL signature was detected in the HPV- HNSCC cohort. This was unexpected, given the positive correlation with NSD1, and the observation that NSD paralogs appear to be expressed in a coordinate fashion regardless of HPV status (Fig. 2).

Damaging mutations in NSD1 and NSD2 were reported to define a subset of stage 3 and 4 laryngeal tumors with favorable prognosis [25]. In addition, mutations in NSD1 are also present more frequently in heavy smokers and correlated with improved overall survival [21]. As mutations of NSD paralogs other than NSD1 are relatively infrequent in the HPV+ TCGA HNSCC cohort [6], we instead chose to assess if NSD1, $N S D 2$, or NSD3 RNA expression was related to overall survival (Fig. 5). A previous report established that NSD1 transcript expression levels are a reasonable estimate of NSD1 proficiency [32]. Therefore, we grouped the HPV+ and HPV- HNSCC cohorts based on high ( $\mathrm{z}-$ score $>0.5)$, mid $(-0.5<\mathrm{z}$-score $<0.5)$, or low (z-score $<$ -0.5 ) expression of each paralog and determined overall survival. Importantly, a dramatic and significant decrease in survival is clearly correlated with reduced NSD1, NSD2, or NSD3 expression in HPV+ samples (Fig. 5a). These survival differences in the NSD1 and NSD3 analyses were independent predictors of survival based on multivariate analysis, with very large effects on relative risk of death (Table 1). It is important to stress that for $\mathrm{HPV}+$ samples, low levels of NSD expression predict poor outcome. In stark contrast, damaging mutations in NSD1 and NSD2 predict a favourable prognosis in HPVHNSCC. Thus, NSDs could potentially serve as prognostic tools in completely different ways in different HNSC $\mathrm{C}$ types. Importantly, despite the obvious potential for using NSD gene expression levels as a tool to identify those HPV+ patients with poor prognosis, NSD levels could also be used as a guide to select those patients that could benefit from treatment de-intensification. For example, those patients in the upper two-thirds of NSD expression exhibit excellent outcomes that may allow less aggressive treatment, while those in the lower third exhibit poor outcomes that may warrant more aggressive treatment.

Given that the NSD paralogs all function as H3K36 methyltransferases, it would seem likely that those HPV+ tumors expressing high NSD levels might exhibit methylation and/or gene expression profiles that differ from those expressing low NSD levels. However, intensive efforts based on hierarchical clustering did not identify HPV+ subsets with different methylomes that predict clinical outcomes [8]. Intriguingly, NSDs also methylate and regulate the activities of other nonhistone substrates, including the proinflammatory NF$\kappa \mathrm{B}$ transcription factor $[15,36]$ and IRF3, a key activator of type-I interferon transcription [17]. As both of these transcription factors play key roles in antiviral immunity, reduced expression in HPV+ HNSCC might limit innate immune responses that help clear these virally induced tumors. This is further supported by the observation that NSD2 knockout impaired the ability of type-I interferon to induce expression of an antiviral gene response [19]. However, NSD levels in HPV+ HNSCC are not correlated with an increased TIL score, suggesting that any potential effects on innate immunity do not correlate with increased infiltration of these already highly infiltrated tumors.

\section{Conclusions}

Taken together, this study provides strong evidence that the NSD methyltransferases play opposite roles within $\mathrm{HPV}+$ and HPV- HNSCC. Most importantly and paradoxically, while mutation/loss of function of NSD members predicts improved clinical outcome in HPV- HNSC $\mathrm{C}$, low level expression of these genes is a very strong predictor of poor outcome in HPV+ HNSCC. Thus, further investigation of the expression levels of NSD1, NSD2, and NSD3 is warranted, as these could serve as rapidly exploitable prognostic biomarkers in HPV + HNSCC, providing a metric for appropriate treatment de-intensification.

\section{Supplementary Information}

The online version contains supplementary material available at https://doi. org/10.1186/s13027-021-00347-6.

\section{Additional file 1}

\section{Abbreviations}

EGFR: Epidermal growth factor receptor; FDR: False discovery rate; H3K4: H3 lysine-4; H4K20: H4 lysine-20; H3K36: H3 lysine-36; HR: Hazard ratio; HNSC 
C: Head and neck squamous cell carcinomas; HPV: Human papillomavirus; HPV-: HPV-negative; HPV+: HPV-positive; IRF3: Interferon regulatory factor 3; NF-kB: Nuclear factor kappa-light-chain-enhancer of activated B cells; NSD: Nuclear receptor binding SET domain protein; PTEN: Phosphatase and tensin homolog; RSEM: RNA-Seq by Expectation-Maximization; TCGA: The Cancer Genome Atlas; TILs: Tumor-infiltrating lymphocytes

\section{Authors' contributions}

Writing - Original Draft: S.F.G., F.G., and J.S.M.; Writing - Review and Editing: All authors; Conceptualization: J.S.M., A.C.N, S.F.G., and F.G.; Software, Formal Analysis and Visualization: S.F.G, F.G., P.Y.F.Z., N.M. and P.P.; Supervision: C.J.H., A.C.N., J.S.M.; All authors have read and agreed to the published version of the manuscript.

\section{Funding}

This work was supported by grants from the Canadian Institutes of Health Research to JSM and ACN (MOP\#142491; PJT-173496) and ACN (MOP\#340674). SFG was supported in part from a Cancer Research and Technology Transfer studentship.

\section{Availability of data and materials}

The following are available online: Additional file 1- Table S1 HNSCC clinical and molecular characteristics.

\section{Ethics approval and consent to participate}

There was no need for ethical approval as all data in this study were downloaded from public databases (TCGA), and the data processing met the TCGA publication guidelines (https://cancergenome.nih.gov/publications/ guidelines).

\section{Consent for publication}

Not applicable.

\section{Competing interests}

The author reports no conflicts of interest in this work.

\section{Author details}

'Department of Microbiology and Immunology, The University of Western Ontario, London, ON N6A 3K7, Canada. ${ }^{2}$ Department of Surgery, The University of Western Ontario, London, ON N6A 3K7, Canada. ${ }^{3}$ Department of Otolaryngology, Head \& Neck Surgery, The University of Western Ontario, London, ON N6A 3K7, Canada. ${ }^{4}$ Department of Pathology, The University of Western Ontario, London, ON N6A 3K7, Canada. ${ }^{5}$ Department of Oncology, The University of Western Ontario, London, ON N6A 3K7, Canada. 'London Regional Cancer Program, Lawson Health Research Institute, London, ON N6C 2R5, Canada. 'Department of Otolaryngology - Head and Neck Surgery, Schulich School of Medicine \& Dentistry, Western University, Room B3-431A, 800 Commissioners Road East, London, ON N6A 5W9, Canada. ${ }^{8}$ London Regional Cancer Program, 790 Commissioners Rd. East, London, Ontario N6A 4L6, Canada.

Received: 5 November 2020 Accepted: 19 January 2021 Published online: 15 February 2021

\section{References}

1. Bray F, Ferlay J, Soerjomataram I, Siegel RL, Torre LA, Jemal A. Global cancer statistics 2018: GLOBOCAN estimates of incidence and mortality worldwide for 36 cancers in 185 countries. CA Cancer J Clin. 2018;68:394-424.

2. Baxi S, Fury M, Ganly I, Rao S, Pfister DG. Ten years of progress in head and neck cancers. J Natl Compr Cancer Netw. 2012;10:806-10.

3. Hashibe M, Brennan P, Chuang S-C, et al. Interaction between tobacco and alcohol use and the risk of head and neck cancer: pooled analysis in the international head and neck Cancer epidemiology consortium. Cancer Epidemiol Biomark Prev. 2009;18:541-50.

4. Marur S, D'Souza G, Westra WH, Forastiere AA. HPV-associated head and neck cancer: a virus-related cancer epidemic. Lancet Oncol. 2010;11:781-9.

5. Nichols AC, Palma DA, Dhaliwal SS, et al. The epidemic of human papillomavirus and oropharyngeal cancer in a Canadian population. Curr Oncol. 2013;20:212-9.

6. Network TCGAR. Comprehensive genomic characterization of head and neck squamous cell carcinomas. Nature. 2015;517:576-82.
7. Mittal S, Banks L. Molecular mechanisms underlying human papillomavirus E6 and E7 oncoprotein-induced cell transformation. Mutat Res. 2017:772:2335.

8. Papillon-Cavanagh S, Lu C, Gayden T, et al. Impaired H3K36 methylation defines a subset of head and neck squamous cell carcinomas. Nat Genet. 2017;49:180-5.

9. Brennan K, Koenig JL, Gentles AJ, Sunwoo JB, Gevaert O. Identification of an atypical etiological head and neck squamous carcinoma subtype featuring the CpG island methylator phenotype. EBioMedicine. 2017;17:223-36.

10. Ang KK, Harris J, Wheeler R, et al. Human papillomavirus and survival of patients with oropharyngeal cancer. N Engl J Med. 2010;363:24-35.

11. Fakhry C, Westra WH, Li S, Cmelak A, Ridge JA, Pinto H, Forastiere A, Gillison ML. Improved survival of patients with human papillomavirus-positive head and neck squamous cell carcinoma in a prospective clinical trial. J Natl Cancer Inst. 2008:100:261-9.

12. Masterson L, Moualed D, Masood A, Dwivedi RC, Benson R, Sterling JC, Rhodes KM, Sudhoff H, Jani P, Goon P. De-escalation treatment protocols for human papillomavirus-associated oropharyngeal squamous cell carcinoma. Cochrane Database Syst Rev. 2014;47:CD010271.

13. Lucio-Eterovic AK, Singh MM, Gardner JE, Veerappan CS, Rice JC, Carpenter $\mathrm{PB}$. Role for the nuclear receptor-binding SET domain protein 1 (NSD1) methyltransferase in coordinating lysine 36 methylation at histone 3 with RNA polymerase II function. Proc Natl Acad Sci U S A. 2010;107:16952-7.

14. Morishita M, di Luccio E. Cancers and the NSD family of histone lysine methyltransferases. Biochim Biophys Acta. 2011;1816:158-63.

15. Lu T, Jackson MW, Wang B, Yang M, Chance MR, Miyagi M, Gudkov AV, Stark GR. Regulation of NF-kappaB by NSD1/FBXL11-dependent reversible lysine methylation of p65. Proc Natl Acad Sci U S A. 2010;107:46-51.

16. Zhang J, Lee Y-R, Dang F, et al. PTEN methylation by NSD2 controls cellular sensitivity to DNA damage. Cancer Discov. 2019;9:1306-23.

17. Wang C, Wang Q, Xu X, Xie B, Zhao Y, Li N, Cao X. The methyltransferase NSD3 promotes antiviral innate immunity via direct lysine methylation of IRF3. J Exp Med. 2017;214:3597-610.

18. Saloura V, Vougiouklakis T, Zewde M, et al. WHSC1L1-mediated EGFR monomethylation enhances the cytoplasmic and nuclear oncogenic activity of EGFR in head and neck cancer. Sci Rep. 2017;7:40664.

19. Sarai N, Nimura K, Tamura T, Kanno T, Patel MC, Heightman TD, Ura K, Ozato K. WHSC1 links transcription elongation to HIRA-mediated histone H3.3 deposition. EMBO J. 2013;32:2392-406.

20. Schneider R, Bannister AJ, Kouzarides T. Unsafe SETs: histone lysine methyltransferases and cancer. Trends Biochem Sci. 2002;27:396-402.

21. Ghasemi F, Prokopec SD, MacNeil D, et al. Mutational analysis of head and neck squamous cell carcinoma stratified by smoking status. JCl Insight. 2019;4:158.

22. Pan C, Izreig S, Yarbrough WG, Issaeva N. NSD1 mutations by HPV status in head and neck cancer: differences in survival and response to DNAdamaging agents. Cancers Head Neck. 2019;4:3.

23. Bui N, Huang JK, Bojorquez-Gomez A, et al. Disruption of NSD1 in head and neck Cancer promotes favorable chemotherapeutic responses linked to Hypomethylation. Mol Cancer Ther. 2018;17:1585-94.

24. Brennan K, Shin JH, Tay JK, Prunello M, Gentles AJ, Sunwoo JB, Gevaert O. NSD1 inactivation defines an immune cold, DNA hypomethylated subtype in squamous cell carcinoma. Sci Rep. 2017;7:17064.

25. Peri S, Izumchenko E, Schubert AD, et al. NSD1- and NSD2-damaging mutations define a subset of laryngeal tumors with favorable prognosis. Nat Commun. 2017:8:1772

26. Seiwert TY, Zuo Z, Keck MK, et al. Integrative and comparative genomic analysis of HPV-positive and HPV-negative head and neck squamous cell carcinomas. Clin Cancer Res. 2015;21:632-41.

27. Chakravarthy A, Henderson S, Thirdborough SM, Ottensmeier $\mathrm{CH}_{1}$ Su X Lechner M, Feber A, Thomas GJ, Fenton TR. Human papillomavirus drives tumor development throughout the head and neck: improved prognosis is associated with an immune response largely restricted to the oropharynx. J Clin Oncol. 2016;34:4132-41.

28. Liu J, Lichtenberg T, Hoadley KA, et al. An integrated TCGA Pan-Cancer clinical data resource to drive high-quality survival outcome analytics. Cell. 2018:173:400-16 e11.

29. Gameiro SF, Kolendowski B, Zhang A, Barrett JW, Nichols AC, Torchia J, Mymryk JS. Human papillomavirus dysregulates the cellular apparatus controlling the methylation status of H3K27 in different human cancers to consistently alter gene expression regardless of tissue of origin. Oncotarget. 2017;8:72564-76. 
30. Wickham H (2016) ggplot2: elegant graphics for data analysis.

31. Spranger $S$, Bao R, Gajewski TF. Melanoma-intrinsic $\beta$-catenin signalling prevents anti-tumour immunity. Nature. 2015;523:231-5.

32. Mandal R, Şenbabaoğlu Y, Desrichard A, et al. The head and neck cancer immune landscape and its immunotherapeutic implications. JCl Insight. 2016;1:e89829

33. Gameiro SF, Ghasemi F, Barrett JW, Koropatnick J, Nichols AC, Mymryk JS, Vareki SM. Treatment-naiive HPV+ head and neck cancers display a T-cellinflamed phenotype distinct from their HPV- counterparts that has implications for immunotherapy. Oncoimmunology. 2018;7:e1498439.

34. Bratman SV, Bruce JP, O'Sullivan B, Pugh TJ, Xu W, Yip KW, Liu F-F. Human papillomavirus genotype association with survival in head and neck squamous cell carcinoma. JAMA Oncol. 2016;2:823-6.

35. Nichols AC, Dhaliwal SS, Palma DA, et al. Does HPV type affect outcome in oropharyngeal cancer? J Otolaryngol Head Neck Surg. 2013;42:9.

36. Yang P, Guo L, Duan ZJ, Tepper CG, Xue L, Chen X, Kung H-J, Gao AC, Zou JX, Chen H-W. Histone methyltransferase NSD2/MMSET mediates constitutive NF-kB signaling for cancer cell proliferation, survival, and tumor growth via a feed-forward loop. Mol Cell Biol. 2012;32:3121-31.

\section{Publisher's Note}

Springer Nature remains neutral with regard to jurisdictional claims in published maps and institutional affiliations.

Ready to submit your research? Choose BMC and benefit from:

- fast, convenient online submission

- thorough peer review by experienced researchers in your field

- rapid publication on acceptance

- support for research data, including large and complex data types

- gold Open Access which fosters wider collaboration and increased citations

- maximum visibility for your research: over $100 \mathrm{M}$ website views per year

At $\mathrm{BMC}$, research is always in progress.

Learn more biomedcentral.com/submissions 